\title{
DIMENSIONAMIENTO DE SECCIONES DE HORMIGON ARMADO ORIENTADO A GARANTIZAR UN NIVEL DE DUCTILIDAD
}

\author{
(DESIGN OF REINFORCED CONCRETE SECTIONS TO ENSURE A DUCTILITY LEVEL)
}

\author{
Antonio R. Marí Bernat, Dr. Ingeniero de Caminos, Canales y Puertos*
}

\section{RESUMEN}

En este artículo se plantea la conveniencia de garantizar un mínimo de ductilidad en las secciones críticas de pórticos y vigas continuas de hormigón armado, para evitar roturas localizadas bajo niveles de carga inferiores al de proyecto.

\section{Se aborda el estudio analítico de secciones} rectangulares de hormigón armado sometidas a flexión en estado límite último, obteniéndose una relación analítica directa entre la profundidad de la fibra neutra $x / d$ y la diferencia de cuantías mecánicas de armaduras de tracción y compresión.

Por último, se propone un método para el dimensionamiento de armaduras en secciones de hormigón armado que garantice unas condiciones de ductilidad prefijadas, establecidas en términos de profundidad máxima de la fibra neutra o bien de diferencia de cuantías. El método se aplica a algún caso práctico, demostrándose el interés del mismo, ya que se consiguen estructuras mucho más dúctiles sin

incremento de coste alguno y de ejecución más sencilla.

\section{SUMMARY}

The convenience of satisfying a ductility condition at critical sections of reinforced concrete frames and continuos beams in order to avoid local failures under low loading levels is discussed in this paper.

The behaviour of rectangular reinforced concrete sections subjected to bending and axial load in ultimate limit state is studied, and direct analytical relationships between the relative depth of neutral axis $x / d$ and the difference of tension and compression reinforcement mechanical ratios are obtained.

Finally a method for the design of the reinforcement necessary to resist a bending moment with a given ductility level, in terms of $x / d$ or $w-w$ ' is presented. The method is applied to practical cases and its interest is shown since much more ductile structures are designed without increase in the cost and in the construction difficulties.

\section{SOBRE LA CONVENIENCIA DE GARANTIZAR UN NIVEL DE DUCTILIDAD SECCIONAL EN ESTRUCTURAS DE HORMIGON ARMADO}

La ductilidad seccional, entendida cualitativamente como la capacidad de desarrollar deformaciones plásticas cuando las armaduras han alcanzado el límite elástico, es una propiedad generalmente poco exigida, en nuestro país, en el dimensionamiento de secciones de hormigón armado. Esta falta de preocupación queda manifiesta especialmente en algunas estructuras de edificación, como es el caso de forjados con jáce-

\footnotetext{
* Profesor Titular. Cátedra de Hormigón. E.T.S. Ing. de Caminos. Universitat Politècnica de Catalunya.
}

nas planas, $u$ otros casos en los que se proyectan elementos a flexión con reducido canto y grandes cuantías mecánicas de armadura. Ello da lugar a un comportamiento estructural poco deseable, que incluso podría ocasionar roturas de tipo frágil, lo cual debe evitarse, entre otras por las razones siguientes:

1.-La rotura frágil no «avisa», ya que el acero no ha llegado a plastificar y, por tanto, no existen deformaciones muy marcadas ni tanta fisuración como en caso de rotura dúctil.

2.- La capacidad de disipación de energía es muy limitada, por lo que en zonas sísmicas deben evitarse totalmente dimensionamientos supracríticos. 
3.-Cuando una sección rompe frágilmente, dada su escasa capacidad de rotación plástica, no permite la redistribución suficiente de esfuerzos ni la acomodación de deformaciones impuestas en estructuras hiperestáticas.

4.-No se aprovecha suficientemente el acero, ya que éste trabaja a tensiones reducidas.

5.-En situaciones de servicio, el importante bloque de compresiones en el hormigón da lugar, en estos casos, a considerables deformaciones por fluencia.

Atendiendo a las consideraciones anteriores parece claro que debería establecerse'una condición de ductilidad a satisfacer en el dimensionamiento de secciones. Ahora bien, ¿qué parámetros pueden utilizarse para conseguir tal nivel de ductilidad?, ¿qué repercusión en el coste de la estructura puede tener el cumplimiento de tal condición?

El parámetro generalmente más aceptado para medir el nivel de ductilidad es la profundidad, relativa al canto útil, de la fibra neutra de la sección en rotura, $x / d$, de forma que las secciones más dúctiles son aquéllas con $\mathrm{x} / \mathrm{d}$ menor. Este parámetro tiene la ventaja de ser muy general (puede utilizarse independientemente de la forma de la sección, de la existencia o no de esfuerzo axil, del tipo de acción -directa o indirecta-y de la presencia o no de pretensado). Sin embargo, tiene el inconveniente de ser una magnitud que el proyectista no suele manejar directamente al dimensionar. Parecería más lógico utilizar algún parámetro de tipo geométrico o mecánico (dimensiones, resistencias o cuantías de armaduras) de fácil manejo en el proyecto, como variable de control de la ductilidad. El objeto de la primera parte de este trabajo es identificar la variable de proyecto a utilizar a tal fin.

En cuanto al segundo interrogante planteado, se proporciona una respuesta en la segunda parte de este artículo, donde se propone un procedimiento para dimensionar las armaduras de secciones rectangulares de hormigón armado, en estado límite último de flexocompresión, para garantizar un nivel determinado de ductilidad y se estudia el coste total de la sección en función del nivel de ductilidad requerido.

\section{COMPORTAMIENTO SECCIONAL EN ROTURA: PROFUNDIDAD $x / d$ Y CUANTIAS DE ARMADURA}

\subsection{Dominios de rotura en hormigón armado}

La rotura de una sección de hormigón armado se define, convencionalmente, por los dominios de deformación (Fig. 2.1.).

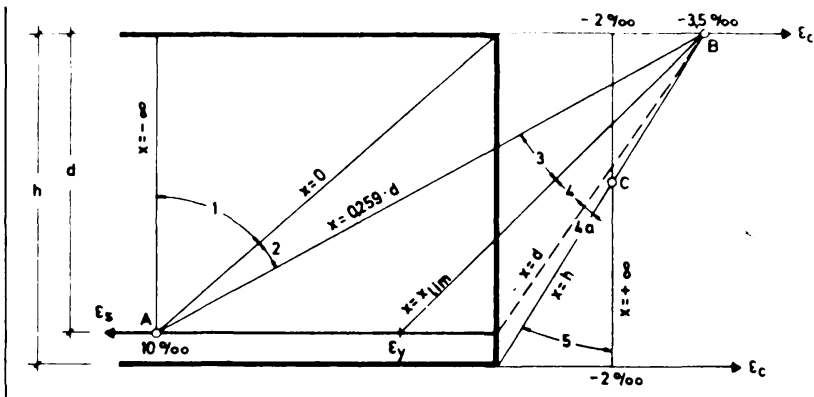

Fig. 2.1.-Dominios de deformación en ELU.

Cuando la sección está sometida a flexión simple, la rotura puede producirse en los dominios 2 , 3 y 4 . El plano frontera entre los dominios 3 y 4 (plano en el que $\varepsilon_{\mathrm{c}}=0,0035, \varepsilon_{\mathrm{s}}=\varepsilon_{\mathrm{y}}$ ) es el que separa la rotura dúctil de la frágil. (Dominios 2 y 3 son dúctiles, dominio 4 , con $\varepsilon_{s}<\varepsilon_{y}$, es frágil.)

\section{2.-Ecuaciones de equilibrio seccional en flexión pura}
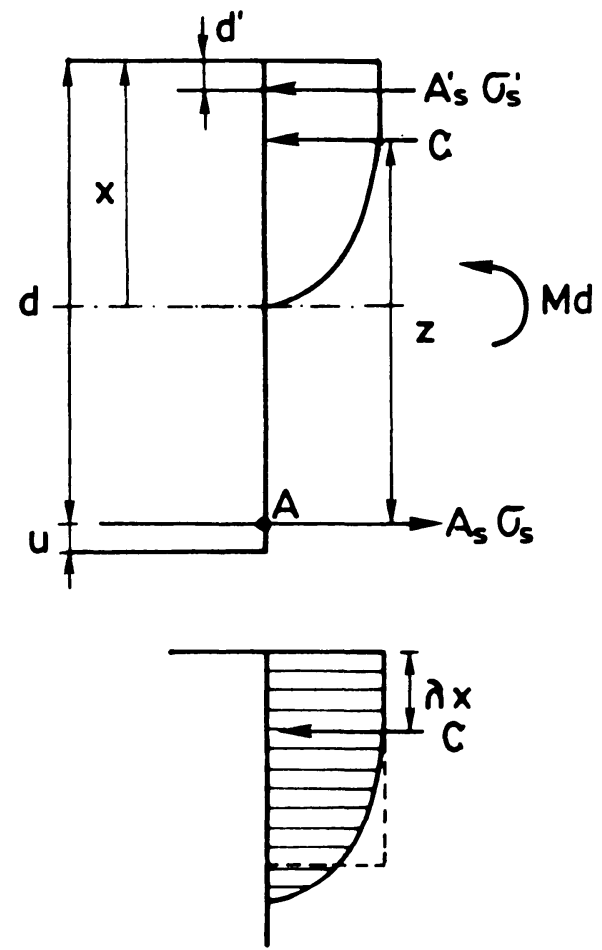

Fig. 2.2.-Esfuerzos y tensiones en la sección.

$$
\begin{array}{cc}
\mathrm{O}=\mathrm{C}+\mathrm{A}_{\mathrm{s}}^{\prime} \cdot \sigma_{\mathrm{s}}^{\prime}-\mathrm{A}_{\mathrm{s}} \sigma_{\mathrm{s}} \quad \Sigma \mathrm{F}_{\mathrm{H}}=0 \\
\mathrm{Md}=\mathrm{C} \cdot \mathrm{Z}+\mathrm{A}_{\mathrm{s}}^{\prime} \cdot \sigma_{\mathrm{s}}^{\prime}\left(\mathrm{d}-\mathrm{d}^{\prime}\right) \quad \Sigma \mathrm{M}_{\mathrm{A}}=0
\end{array}
$$


Siendo,

$$
\begin{gathered}
C=\psi f_{c d} \cdot b \cdot x \\
Z=d-\lambda x
\end{gathered}
$$

Los valores de $\psi$ y $\lambda$ dependen de la deformación de la fibra extrema del hormigón en compresión $\varepsilon_{\mathrm{c}^{\prime}}$ y adoptan, por tanto, expresiones diferentes según el dominio de deformación.

En el dominio 2 se pueden distinguir dos casos:

\section{Dominio 2.a}

$$
\begin{gathered}
-0,002<\varepsilon_{\mathrm{c}} \leqq 0, \text { o bien } 0 \leqq \xi \leqq 0,1667, \xi=\frac{x}{\mathrm{~d}} \\
\psi=\frac{4,25 \xi(3-8 \xi)}{3(1-\xi)^{2}} \\
\lambda=\frac{4-9 \xi}{12-32 \xi}
\end{gathered}
$$

\section{Dominio 2.b.}

$-20,002>\varepsilon_{c} \geqq-0,0035$, o bien $0,1667 \leqq \xi<0,2593$

$$
\begin{gathered}
\psi=0,85 \frac{16 \xi-1}{15 \xi} \\
\lambda=\frac{171 \xi^{2}-22 \xi+1}{320 \xi^{2}-20 \xi}
\end{gathered}
$$

\section{Dominios 3 y 4}

Dado que $\varepsilon_{\mathrm{c}}=0,0035$ en todos los planos de rotura, $\lambda$ y $\psi$ permanecen constantes e iguales a:

$$
\begin{aligned}
& \psi=0,6881 \\
& \lambda=0,4160
\end{aligned}
$$

Los valores de $\psi$ y $\lambda$ en función de $\xi$ quedan reflejados en la tabla 2.1, extraída directamente de la referencia 8.

Puestas en forma adimensional las ecuaciones de equilibrio quedan así (dividiendo la primera por $f_{c d} \cdot b \cdot d$ y la segunda por $\left.f_{c d} \cdot b \cdot d^{2}\right)$ :

$$
\mathrm{O}=\psi \cdot\left(\frac{\mathrm{x}}{\mathrm{d}}\right)+\mathrm{w}^{\prime} \cdot \frac{\sigma_{\mathrm{s}}^{\prime}}{\mathrm{f}_{\mathrm{yd}}}-\mathrm{w} \cdot \frac{\sigma_{\mathrm{s}}}{\mathrm{f}_{\mathrm{yd}}}
$$

$$
\begin{aligned}
& \mu_{d}=\psi \cdot\left(\frac{x}{d}\right) \cdot\left[1-\lambda\left(\frac{x}{d}\right)\right]+ \\
&+w^{\prime} \cdot\left(1-\frac{d^{\prime}}{d}\right)
\end{aligned}
$$

donde,

$\mu_{d}=\frac{M d}{f_{c d} \cdot b \cdot d^{2}} \begin{aligned} & \text { Momento relativo o adi- } \\ & \text { mensional }\end{aligned}$

$w=\frac{A_{s} \cdot f_{y d}}{f_{c d} \cdot b \cdot d}$ Cuantía mecánica de ar-

$w^{\prime}=\frac{A_{s}^{\prime} \cdot f_{y d}}{f_{c d} \cdot b \cdot d} \begin{gathered}\text { Cuantía mecánica de ar- } \\ \text { madura de compresión }\end{gathered}$

TABLA 2.1

Valores de $\psi$ y $\lambda$ en el dominio 2

\begin{tabular}{|c|c|c|}
\hline$\xi$ & $\psi$ & $\lambda$ \\
\hline 0,0800 & 0,31601 & 0,34746 \\
0,0900 & 0,35104 & 0,34978 \\
0,1000 & 0,38477 & 0,35227 \\
0,1100 & 0,41708 & 0,35495 \\
0,1200 & 0,44783 & 0,35784 \\
0,1300 & 0,47690 & 0,36097 \\
0,1400 & 0,50415 & 0,36436 \\
0,1500 & 0,52941 & 0,36806 \\
0,1600 & 0,55253 & 0,37209 \\
0,1667 & 0,56673 & 0,37502 \\
\hline 0,1700 & 0,57333 & 0,37652 \\
0,1800 & 0,59185 & 0,38126 \\
0,1900 & 0,60842 & 0,38611 \\
0,2000 & 0,62333 & 0,39091 \\
0,2100 & 0,63683 & 0,39559 \\
0,2200 & 0,64909 & 0,40011 \\
0,2300 & 0,66029 & 0,40444 \\
0,2400 & 0,67056 & 0,40857 \\
0,2500 & 0,68000 & 0,41250 \\
0,2590 & 0,68788 & 0,41587 \\
\hline 0,2593 & 0,6881 & 0,416 \\
\hline
\end{tabular}

\subsection{Valor de la profundidad de la fibra neutra en rotura}

De la ecuaciớn de equilibrio de fuerzas, despejando $\frac{x}{d}$ se tiene:

$\frac{x}{d}=\frac{w \cdot \frac{\sigma_{s}}{f_{y d}}-w^{\prime} \cdot \frac{\sigma_{s}}{f_{y d}}}{\psi}$ 
Esta expresión, que es totalmente general y válida para los dominios 2, 3 y 4, adopta formas muy sencillas en los dominios $2 b$ y 3 , como se verá a continuación:

En el dominio 2.a

$$
\sigma_{\mathrm{s}}=f_{\mathrm{yd}} \quad\left(\varepsilon_{\mathrm{s}}=0,01\right)
$$

$$
\xi=\frac{w-w^{\prime} \cdot \frac{\sigma_{s}^{\prime}}{f_{y d}}}{\psi}
$$

$\sigma_{s}^{\prime}=\varepsilon_{s}^{\prime} \cdot E_{s}=0,01 \cdot \frac{x-d^{\prime}}{d-x} \cdot E_{s}$

$\psi$ adopta el valor de la ecuación [2.7].

\section{Dominio 2.b}

$\sigma_{\mathrm{s}}=\mathrm{f}_{\mathrm{yd}} \quad\left(\varepsilon_{\mathrm{s}}=0,01\right)$

Se puede adoptar sin demasiado error $\sigma_{s}^{\prime}=f_{y d}$, si el recubrimiento d' no es muy grande, o sea, si $d^{\prime} \leqq 0,04 d$.

$$
\frac{x}{d}=\frac{w-w}{\psi}
$$

donde $\psi$ adopta el valor de la ecuación [2.9].

Se puede despejar directamente $\frac{x}{d}$ en función de $w-w^{\prime}$

$$
\frac{x}{d}=1,103\left(w-w^{\prime}\right)+0,0625
$$

con el intervalo de validez $0,095 \leqq w-w^{\prime} \leqq 0,1784$.

\section{Dominio 3}

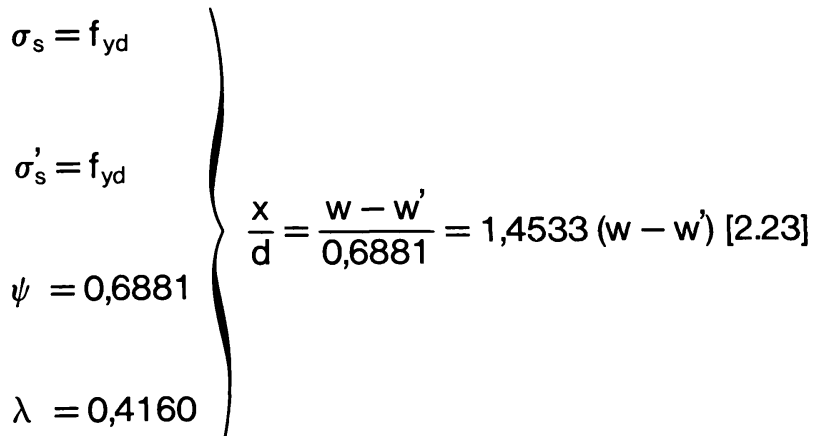

Dominio 4

$$
\begin{aligned}
& \left.\begin{array}{l}
\sigma_{s}=E_{s} \cdot \varepsilon_{s}=0,0035\left(\frac{d}{x}\right) E_{s} \\
\sigma_{s}^{\prime} f_{y d} \\
\begin{array}{l}
\psi=0,6881 \\
\lambda=0,4160
\end{array}
\end{array}\right\}=\frac{w \cdot \frac{\sigma_{s}}{f_{y d}}-w^{\prime}}{0,6881} \\
& {[2.24]}
\end{aligned}
$$

Haciendo:

$$
\alpha=\frac{0,0035 \cdot E_{s}}{f_{y d}}
$$

$\frac{x}{d}=\frac{-\left(w \alpha+w^{\prime}\right)+\sqrt{\left(w \alpha+w^{\prime}\right)^{2}+2.752 w \alpha}}{1.376}$

\subsection{Caracterización del tipo de rotura seccional}

Para saber si la rotura de la sección será dúctil o frágil, basta comparar el valor de $\frac{x}{d}$ con la profundidad límite, es decir, aquella para la cual el acero tiene una deformación $\varepsilon_{\mathrm{s}}=\varepsilon_{\mathrm{y}}$ y el hormigón $\varepsilon_{\mathrm{c}}=-0,0035$, cuyo valor es:

$$
\left(\frac{x}{d}\right)_{\lim }=\frac{1}{1+1,36 \cdot 10^{-4} \cdot f_{y d}}
$$

Para ese plano, por estar justo en la separación entre los dominiss 3 y 4 se tiene que $\psi=0,6881$, $\sigma_{s}=\sigma_{s}^{\prime}=f_{y d}, \frac{x}{d}=\frac{w-w^{\prime}}{0,6881}$. Por tanto, la condición de rotura dúctil será $\frac{x}{d} \leqq\left(\frac{x}{d}\right)_{\lim }$ es decir,

$$
\begin{aligned}
\frac{w-w^{\prime}}{0,6881} & \leqq \frac{1}{1+1,36 \cdot 10^{-4} \cdot f_{y d}} \\
\left(w-w^{\prime}\right) & \leqq \frac{0,6881}{1+1,36 \cdot 10^{-4} \cdot f_{y d}}
\end{aligned}
$$

La tabla 2.2 presenta los valores de $\left(w-w^{\prime}\right)_{\text {lim }}$ para diversos casos frecuentes de acero.

TABLA 2.2

Valores de profundidad y cuantías límites

\begin{tabular}{|l|c|c|c|c|}
\hline Acero & AEH 400N; & $f_{y k}=4100$ & AEH 500N; & $f_{y k}=5100$ \\
\hline Coefic. & $\gamma_{s}=1,15$ & $\gamma_{s}=1,1$ & $\gamma_{s}=1,15$ & $\gamma_{s}=1,10$ \\
\hline$\left(\frac{x}{d}\right)_{\lim }$ & 0,6734 & 0,6630 & 0,6240 & 0,6133 \\
\hline$\left(w-w^{\prime}\right)_{\lim }$ & 0,4634 & 0,4566 & 0,4293 & 0,4220 \\
\hline
\end{tabular}




\section{DIMENSIONAMIENTO DE LAS ARMADURAS PARA UN NIVEL DE DUCTILIDAD PREFIJADO}

Tal como se ha comentado en la introducción, el parámetro generalmente aceptado para medir la ductilidad es la relación $\frac{x}{d}$ en rotura. En el apartado 2 se ha visto que, para secciones rectangulares y otras asimilables en rotura, la profundidad $\frac{x}{d}$ está directamente relacionada con la diferencia de cuantías $w-w$, por lo que es posible limitar, mediante la adecuada disposición de armaduras de compresión y de tracción, el valor de $\frac{x}{d}$ a uno prefijado.

El valor de $\frac{x}{d}$ máximo viene, generalmente, establecido en las Normativas, por razones de tipo sísmico y el grado de redistribución de esfuerzos adoptado para el dimensionamiento. Macchi (1) propone no superar el valor $\frac{x}{d}=0,45 \mathrm{ni}$ siquiera utilizando un cálculo lineal.

En este apartado se describe el procedimiento de obtención de las cuantías mecánicas $w$ y w' de manera que, además de resistir el momento que solicita la sección se satisfaga la condición de ductilidad prefijada.

Las ecuaciones a tener en cuenta son:

1) Condición de ductilidad prefijada

$$
\frac{x}{d} \leqq\left(\frac{x}{d}\right)_{\text {máx }}
$$

2) Ecuación de equilibrio de fuerzas, válida para todos los dominios

$$
\frac{x}{d}=\frac{w \cdot \frac{\sigma_{s}}{f_{y d}}-w^{\prime} \cdot \frac{\sigma_{s}^{\prime}}{f_{y d}}}{\psi}
$$

3) Ecuación de equilibrio de momentos. $\mu_{c}$ es el Momento proporcionado por las tensiones de compresión en el hormigón

$$
\begin{aligned}
& \mu_{d}=\psi \cdot\left(\frac{x}{d}\right)\left(1-\lambda \cdot \frac{x}{d}\right)+w^{\prime}\left(1-\frac{d^{\prime}}{d}\right)= \\
& =\mu_{c}+w^{\prime} \cdot\left(1-\frac{d^{\prime}}{d}\right)
\end{aligned}
$$

El procedimiento a seguir es el siguiente:

1) Se define el momento límite como aquel que puede ser resistido, sin armadura de compre(c) Consejo Superior de Investigaciones Científicas Licencia Creative Commons 3.0 España (by-nc) sión, siendo $\left(\frac{x}{d}\right)=\left(\frac{x}{d}\right)_{\text {máx }}$. De la ecuación de equilibrio de momentos [2.14] se tiene:

$\mu_{\text {lim }}=\psi \cdot\left(\frac{x}{d}\right)_{\text {máx }}\left[1-\lambda \cdot\left(\frac{x}{d}\right)_{\text {máx }}\right]$

Los valores de $\psi$ y $\lambda$ son conocidos, pues se conoce $\left(\frac{x}{d}\right)_{\text {máx }} y$, por tanto, el dominio correspondiente (ver tabla 2.1).

2) Para momentos $\mu_{d}$ inferiores al momento límite, no hace falta armadura de compresión $\left(w^{\prime}=0\right)$ y la armadura de tracción se calcula de la misma manera que se haría sin condición de ductilidad alguna.

3) Para momentos $\mu_{d}$ superiores al momento límite se debe colocar armadura de compresión w' que absorba el exceso de momento, de manera que:

$$
\begin{gathered}
\mu_{c}=\mu_{\lim } \text { y }\left(\frac{x}{d}\right)=\left(\frac{x}{d}\right)_{\text {máx }} \\
\mu_{d}=\mu_{\lim }+w^{\prime} \cdot\left(1-\frac{d^{\prime}}{d}\right)
\end{gathered}
$$

de donde

$$
\begin{aligned}
& w^{\prime}=\frac{\mu_{d}-\mu_{\text {lim }}}{1-\frac{d^{\prime}}{d}} \\
& w=\psi \cdot\left(\frac{x}{d}\right)_{\text {máx }}^{d}+w^{\prime}=\psi \cdot\left(\frac{x}{d}\right)_{\text {máx }}^{+} \\
& +\frac{\mu_{d}-\mu_{\text {lim }}}{1-\frac{d^{\prime}}{d}}
\end{aligned}
$$

donde todos los valores son conocidos. En este caso, para la obtención de w en la ecuación de equilibrio de axiles se hace $\sigma_{s}=\sigma_{s}^{\prime}=f_{y d}$ con lo que se aprovechan al máximo las armaduras.

En definitiva, lo que se hace es variar el momento a partir del cual se necesita armadura de compresión (momento límite), en función del máximo valor de $\frac{\mathrm{x}}{\mathrm{d}}$ permitido por la condición de ductilidad. La figura y tabla 3.1 presentan $\mu_{\text {lim }}$ en función de $\left(\frac{x}{d}\right)_{\text {máx }}$. Obsérvese que limitar, por ejemplo, el valor $\frac{x}{d}$ a 0,45 equivale a limitar el momento adimensional a 0,252 si no se utiliza armadura de compresión.

http://informesdelaconstruccion.revistas.csic.es 
TABLA 3.1

Momento límite adimensional

\begin{tabular}{|c|c|c|c|}
\hline$\frac{\mathbf{x}}{\mathbf{d}}$ & $\mathbf{M}$. Limite & $\frac{\mathbf{x}}{\mathbf{d}}$ & $\mathbf{M}$. Límite \\
\hline 0,080 & 0,0246 & 0,380 & 0,220 \\
0,090 & 0,0306 & 0,390 & 0,225 \\
0,100 & 0,0371 & 0,400 & 0,229 \\
0,110 & 0,0441 & 0,410 & 0,234 \\
0,120 & 0,0514 & 0,420 & 0,239 \\
0,130 & 0,0591 & 0,430 & 0,243 \\
0,140 & 0,0670 & 0,440 & 0,247 \\
0,150 & 0,0750 & 0,450 & 0,252 \\
0,160 & 0,0831 & 0,460 & 0,256 \\
0,170 & 0,0912 & 0,470 & 0,260 \\
0,180 & 0,0992 & 0,480 & 0,264 \\
0,190 & 0,1071 & 0,490 & 0,268 \\
0,200 & 0,1149 & 0,500 & 0,272 \\
0,210 & 0,1226 & 0,510 & 0,276 \\
0,220 & 0,1302 & 0,520 & 0,280 \\
0,230 & 0,1377 & 0,530 & 0,284 \\
0,240 & 0,1452 & 0,540 & 0,288 \\
0,250 & 0,1525 & 0,550 & 0,292 \\
0,260 & 0,1596 & 0,560 & 0,296 \\
0,270 & 0,1649 & 0,570 & 0,299 \\
0,280 & 0,1702 & 0,580 & 0,303 \\
0,290 & 0,1755 & 0,590 & 0,306 \\
0,300 & 0,1807 & 0,600 & 0,310 \\
0,310 & 0,1858 & 0,610 & 0,313 \\
0,320 & 0,1909 & 0,620 & 0,317 \\
0,330 & 0,1959 & 0,630 & 0,320 \\
0,340 & 0,2009 & 0,640 & 0,323 \\
0,350 & 0,2058 & 0,650 & 0,326 \\
0,360 & 0,2106 & 0,660 & 0,329 \\
0,370 & 0,2154 & 0,670 & 0,333 \\
\hline
\end{tabular}

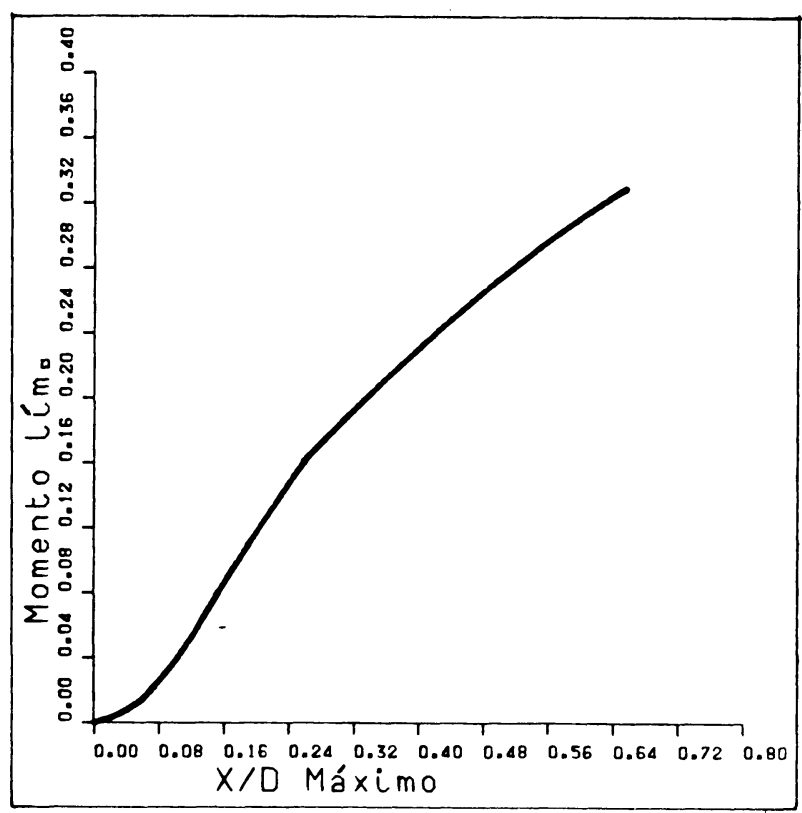

Fig. 3.1.-Momento límite adimensional en función de $x / d$.

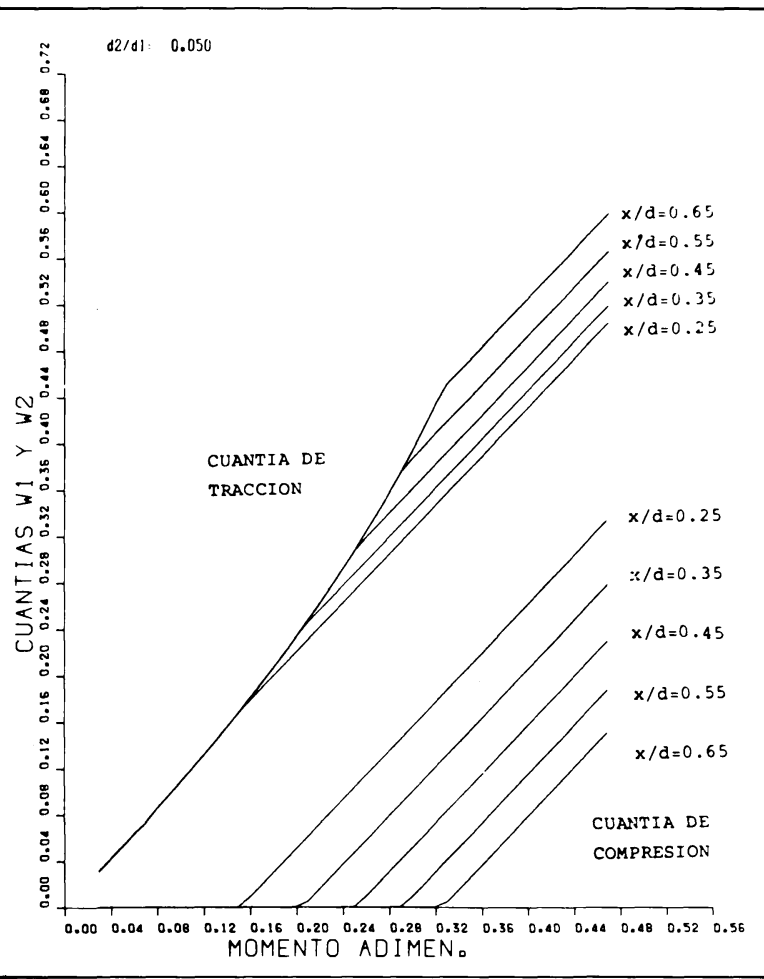

Fig. 3.2.-Abaco de dimensionamiento de armaduras para un valor de $x / d$ prefijado.

Mediante aplicación del procedimiento antes descrito pueden obtenerse curvas y tablas para dimensionamiento directo de armaduras de compresión y tracción, dado un valor máximo de la profundidad $\frac{x}{d}$ y el momento adimensional a resistir, como las que se presentan en la figura 3.2, la cual corresponde a una relación $\frac{d^{\prime}}{d}=0,05$.

Como se observa en la figura 3.2, a menor valor de $\frac{x}{d}$ (mayor exigencia de ductilidad), para resistir un momento determinado se necesita más armadura de compresión y menos de tracción ( $w-w$ ' es menor).

En la referencia 12 se presenta una gama más completa de tablas y ábacos, para diversos valores de $\frac{x}{d}$ y de relaciones $\frac{d^{\prime}}{d}$.

Ahora bien, ¿hasta qué punto la limitación de la profundidad $\frac{x}{d}$ puede significar un aumento importante en la cuantía total de la sección (y, por tanto, en su coste)? Para responder a esta cuestión observemos la figura 3.3 que representa la cuantía total de armadura $(w+w)$ necesaria para resistir un momento dado ( $\mu=$ constante), con diversas limitaciones de $\frac{x}{d}$. De la figura se de- 


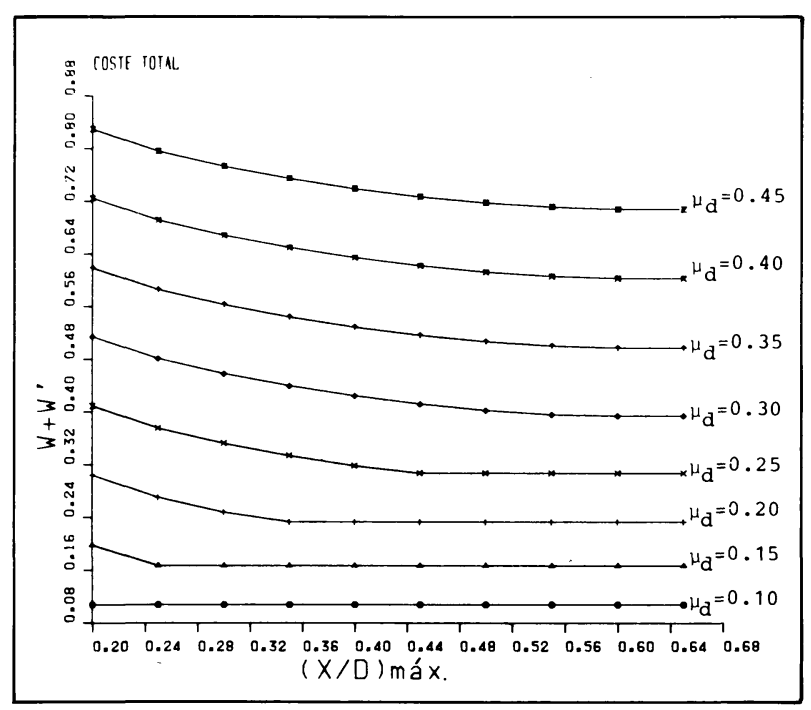

Fig. 3.3.-Cuantía teórica total en función de $x / d$.

duce que la variación de $(w+w$ ') es, en general, muy pequeña; lógicamente a medida que $\left(\frac{x}{d}\right)_{\text {máx }}$ es menor, la cuantía total aumenta, pero muy suavemente. Por ejemplo, en el caso de dimensionamiento frente a un momento $\mu=0,30$, el incremento de cuantía total que supone limitar $\left(\frac{x}{d}\right)$ a 0,45 , respecto de un dimensionamiento sin limitación alguna es de un $4,5 \%$.

En cuanto a las repercusiones a nivel pieza completa, teniendo en cuenta la prolongación de armaduras necesarias por longitudes de anclaje, el hecho de uniformizar las cuantías de tracción y compresión es favorable no tan sólo por razones económicas, sino por simplicidad en la ejecución y racionalización del armado.

En efecto, considérese un pórtico plano de un edificio, constituido por vigas planas y pilares, como se indica en la figura 3.4. De dicha estructura se extrae, para este ejemplo, una viga de $6 \mathrm{~m}$ de luz que recibe una carga característica de $2,81 \mathrm{t} / \mathrm{m}$. El cálculo lineal de la estructura global proporciona la ley de Momentos flectores de la figura, con Momentos de 12, 5,36 y $18 \mathrm{mxt}$ en las secciones $A, B$ y $C$, respectivamente. La viga consiste en una jácena plana de sección rectangular, de 0,60 ×0,30 m.

Para el dimensionamiento de las armaduras se adoptan las propiedades de los materiales indicados en la figura 3.4 .

Se pretende establecer una comparación entre las soluciones obtenidas estableciendo dos requerimientos de ductilidad diferentes en el armado de las secciones más desfavorables, par-
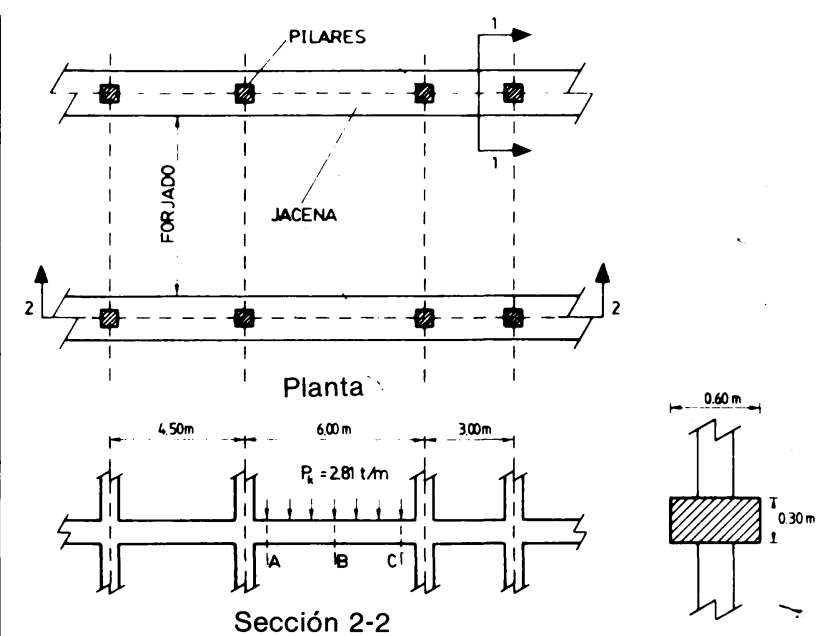

Sección 1-1

ESQUEMA ESTRUCTURAL DEL EJEMPLO

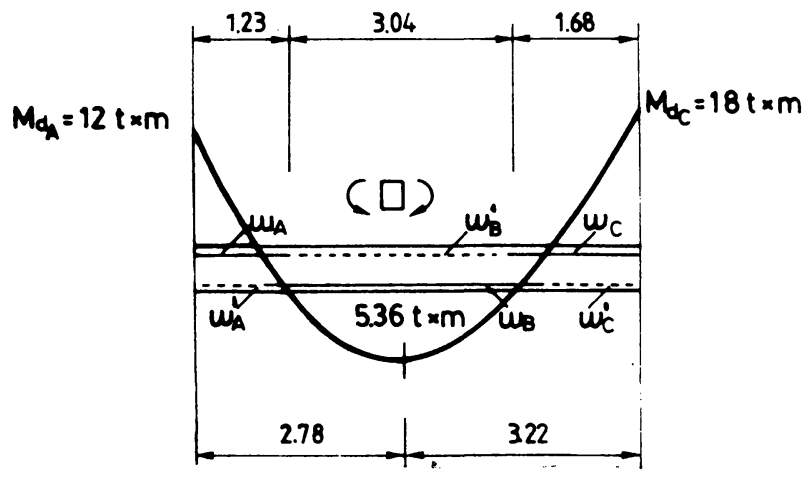

Hormigón $\mathrm{H}-175 \mathrm{f}_{\mathrm{ck}}=175 \mathrm{kp} / \mathrm{cm}^{2}$

Acero AEH-400N $\mathrm{f}_{\mathrm{yk}}=4.100 \mathrm{kp} / \mathrm{cm}^{2}$

$\gamma_{\mathrm{c}}=1,5, \gamma_{\mathrm{s}}=1,1, \gamma_{\mathrm{f}}=1,6$

Fig. 3.4.-Estructura estudiada.

tiendo en ambos casos de un cálculo lineal elástico. En el primer caso no se establece la condición de ductilidad usual, es decir,

$\left(\frac{x}{d}\right) \leqq\left(\frac{x}{d}\right)_{\lim }$ (usualmente 0,65 ). En el segundo caso se limita $\frac{x}{d}$ a 0,45 .

En el cálculo de la armadura de tracción en la primera hipótesis se ha considerado la existencia de armadura de compresión por razones de montaje.

Los resultados del armado en ambas hipótesis se presentan en la tabla 3.2, en la cual se muestran las cuantías en las tres secciones críticas, así como el "coste» total aproximado en términos del producto de las cuantías por las longitudes de cada tramo de armado. 
TABLA 3.2

Resultado del armado con y sin condición de ductilidad

\begin{tabular}{|c|c|c|c|c|c|c|c|c|c|c|c|c|c|}
\hline \multirow[b]{2}{*}{ HIPOTESIS } & \multicolumn{4}{|c|}{ SECCION A } & \multicolumn{4}{|c|}{ SECCION B } & \multicolumn{4}{|c|}{ SECCION C } & \multirow{2}{*}{$\frac{\text { C. TOTAL }}{\sum w_{i} 1_{i}+w_{i} 1_{i}^{\prime}}$} \\
\hline & $\mu \mathbf{A}$ & $w_{A}$ & w'A & $w_{A}+w_{A}^{\prime}$ & $\mu_{\mathrm{d}}^{\mathbf{B}}$ & $w_{B}$ & $w_{B}^{\prime}$ & $w_{B}+w_{B}^{\prime}$ & $\mu \mathrm{d}$ & $w \approx$ & w' & $w_{C}+w_{c}^{\prime}$ & \\
\hline $\begin{array}{l}\text { 1. Cálculo lineal sin } \\
\text { condición de ducti- } \\
\text { lidad }\end{array}$ & 0,228 & 0,261 & 0,04 & 0,301 & 0,102 & 0,108 & 0,04 & 0,148 & 0,342 & 0,445 & 0,04 & 0,485 & 2,240 \\
\hline $\begin{array}{l}\text { 2. Cálculo lineal con } \\
\text { condición } \\
x / d \leqq 0,45\end{array}$ & 0,228 & 0,261 & 0,04 & 0,301 & 0,102 & 0,108 & 0,04 & 0,148 & 0,342 & 0,409 & 0,10 & 0,509 & 2,210 \\
\hline
\end{tabular}

Puede observarse que el coste total de la pieza para las dos soluciones planteadas es sensiblemente constante, habiendo mejorado en el segundo caso la capacidad de adaptación plástica y disipación de energía de la estructura.

\section{CONCLUSIONES}

De lo expuesto en este trabajo se pueden extraer las siguientes conclusiones:

- La diferencia de cuantías mecánicas de compresión y tracción $\left(w-w^{\prime}\right)$ es un parámetro que define el comportamiento en rotura de secciones rectangulares de hormigón armado, en el intervalo $0,095 \leqq w-w^{\prime} \leqq 0,45$ aproximadamente.

- Conocido $w$ - w' es posible conocer la forma y el dominio de rotura, la profundidad de la fibra neutra y la curvatura última de la sección, mediante expresiones analíticas sencillas.

- La diferencia w - w' es, por tanto, un parámetro que regula la ductilidad seccional y que puede ser utilizado con facilidad por el proyectista para dimensionar secciones con capacidad de rotación plástica suficiente para garantizar un nivel de redistribución de esfuerzos. Cuanto menos es $w-w^{\prime}$ más dúctil es la sección.

- Se ha desarrollado un procedimiento simple y preciso para el armado de secciones rectangulares en flexión simple, condicionado a un valor máximo de la profundidad relativa de la fibra neutra.

- El establecimiento de una limitación de la profundidad $x / d$ (o en su caso de $w-w$ ) conduciría al dimensionamiento de secciones y estructuras con un nivel de ductilidad deseado $\sin$ incremento apreciable en el coste total de la estructura e incluso con una mejora de las condiciones de ejecución de la misma.

\section{REFERENCIAS}

1. MACCHI, G.: «Ductility Condition for Simplified Design Without Check of Compatibility». C.E.B. Bulletin d'Information n. 105 . "Structures Hiperestatiques». Febrero 1975.

2. MANFRON, V.; MATTARUCCO, A., y SIVIERO, E.: "On the Ductility Conditions for T-Beams with Variable Spans and Loading Patterns». C.E.B. Bulletin d'Information n. 105 . "Structures Hiperestatiques». Febrero 1975.

3. SIVIERO, E.: "Rotation Capacity of Monodimensional Members in Structural Concrete». C.E.B. Bulletin d'Information n.० 105. "Structures Hiperestatiques". Febrero 1975.

4. MACCHI, G.: «Preliminary Design of Concrete Frames According to the Limit States Method". Instituto Universario Architettura di Venezia, n. ${ }^{\circ}$ 14, 1970.

5. GOSH, S. K. y COHN, M. Z.: "Ductility of Reinforced Concrete Sections in Combined Bending and Axial Load". Inelasticity and Nonlinearity in Structural Concrete. Study n. 8. Solid Mechanics Division. University of Waterloo, Waterloo, Ontario, Canada.

6. APARICIO, A.: «Sobre la conveniencia de limitar, por consideraciones de ductilidad, la cuantía máxima de armaduras de flexión en vigas continuas de hormigón armado y pretensado". Hormigón y Acero n. $144,1982$.

7. CALAVERA, J.: «Proyecto y Cálculo de Estructuras de Hormigón Armado para edificios». Ed. Intemac, Madrid 1984.

8. JIMENEZ MONTOYA, P.; GARCIA MESSEGUER, A., y MORAN CABRE, F.: "Hormigón Armado». Ed. Gustavo Gili.

9. COMISION PERMANENTE DEL HORMIGON: «Instrucción EH-82 para el proyecto y ejecución de estructuras de hormigón en masa y armado". Ministerio de Obras Públicas y Urbanismo (MOPU), 1982.

10. COMITÉ-EURO INTERNATIONAL DU BETÓN: FEDERATION INTERNATIONALE DE LA PRECONTRAINTE CEB-FIP Model Code for Concrete

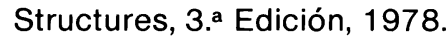

11. MORAN CABRE, F.: «Programa para el dimensionamiento de secciones rectangulares de hormigón armado en flexocompresión recta con un ordenador portátil». Hormigón y Acero n. 153, n. 4, Trimestre de 1984.

12. MARI BERNAT, A. R.: "Cuantías de armaduras y redistribución de esfuerzos en elementos a flexión de hormigón armado y pretensado". Publicación ETS Ingenieros de Caminos de Barcelona. Enero de 1987. 